\title{
Bacillus Calmette Guérin in bladder cancer: is more immune stimulation better?
}

\author{
Mar Valés-Gómez \\ Department of Immunology and Oncology, National Centre for Biotechnology, CNB-CSIC, Darwin, Madrid, Spain \\ Correspondence to: Mar Valés-Gómez. Department of Immunology and Oncology, National Centre for Biotechnology, CNB-CSIC, Darwin, 3, 28049 \\ Madrid, Spain. Email: mvales@cnb.csic.es. \\ Provenance: This is an invited article commissioned by the Section Editor Dr. Xiao Li (Department of Urology, Jiangsu Cancer Hospital \& Jiangsu \\ Institute of Cancer Research \& Affiliated Cancer Hospital of Nanjing Medical University, Nanjing, China). \\ Comment on: Rodriguez D, Goulart C, Pagliarone AC, et al. In vitro Evidence of Human Immune Responsiveness Shows the Improved Potential of a \\ Recombinant BCG Strain for Bladder Cancer Treatment. Front Immunol 2019;10:1460.
}

Submitted Oct 03, 2019. Accepted for publication Dec 05, 2019.

doi: $10.21037 /$ tau.2019.12.23

View this article at: http://dx.doi.org/10.21037/tau.2019.12.23

Intravesical instillation of bacillus Calmette Guérin (BCG) has been the therapy of choice for the treatment of highrisk non-muscle-invasive bladder cancer (NMIBC) for several decades, increasing considerably the survival of these patients. For papillary tumours, the instillations are applied as adjuvant after trans-urethral resection of the bladder (TURB); however, carcinoma in situ (CIS) cannot be excised and, in this case, BCG is used without prior surgery. Despite the advantages provided by this treatment, with around $70 \%$ of patients relapse-free after 5 years, remarkable improvements in the outcome of this therapy have not been achieved since the introduction of maintenance instillation cycles (1). This is probably related to two important facts: (I) administration of BCG varies among different hospitals, including bacterial strain, concentration and schedule; (II) the exact mechanism of action of BCG in the elimination of bladder cancer is not completely understood.

To address the first of these issues, clinical trials have been designed to compare treatment routines. Although these clinical trials have been rather scarce and, in many occasions difficult to compare, it seems clear that the most successful regime includes an induction phase of 6 weekly instillations followed by a maintenance cycle which varies in length and calendar, but in general consists of 2 or 3 weekly instillations alternating with rest periods (1). A second group of clinical trials aimed to evaluate the effect of different BCG strains in the elimination of bladder cancer. However, disappointingly, different trial setups and contradictory results do not allow a definitive conclusion on whether the use of different strains strongly affects the efficacy of the BCG treatment (1).

On the other hand, extensive laboratory-based research over the years has tried to unveil the molecular mechanisms of BCG against cancer and so develop novel strategies to improve this therapy. These research efforts have included studies on the interaction between BCG and different components of the immune system, as well as projects involving the generation of engineered BCG variants expressing either cytokines or immune-stimulating molecules. Further, in an attempt to improve efficacy while decreasing BCG adverse effects, the description of new bacterial-based therapies for bladder cancer is under study.

The paper by Rodriguez et al. (2) is part of the efforts to define new BCG strains that might improve the rate of success in the treatment of bladder cancer. This work has the general goal of triggering a more efficient immune response, based on the use of a recombinant BCG strain expressing the detoxified Pertussis toxin derivative, S1 subunit-9K/129G (S1PT), to try and stimulate immune effector cells more potently. The effect of culturing the toxin derivative-expressing BCG in vitro with human peripheral blood mononuclear cells (PBMC) was studied, and the degree of $\mathrm{CD}^{+} \mathrm{T}$ lymphocyte activation, as well as the cytokines produced in the cultures, was evaluated. The authors exposed PBMC from healthy donors to 
mycobacteria using a multiplicity of infection (MOI) of 0.1 , previously described as optimal for in vitro infection of macrophages, and compared the effect of wild type (wt) BCG with that of the engineered BCG-S1PT. Culture supernatants contained more cytokines when exposed to recombinant bacteria than when wt BCG was used, however, the dose did not correlate with the vaccination status of the donors. One striking result is that there is high variation in the amount of cytokines released by each donor and the authors suggest a personalized test for patients undergoing BCG treatment. The release of cytokines in bladder cancer patients treated with BCG can be easily analysed in urine and several studies have identified increased cytokine concentrations after instillations $(3,4)$. In general, when patient urine was analysed early after instillations, $24-48 \mathrm{~h}$, a considerable degree of acute inflammation is still ongoing and pro-inflammatory cytokines such as tumour necrosis factor (TNF)- $\alpha$, IL-6, IL-1 $\beta$, and IL-8 could be detected. Several groups have proposed the use of these soluble factors, alone or in combination with other clinical data, as biomarkers of treatment response. For example, vascular endothelial growth factor (VEGF) (5), IL-8 (6-8) or the nomogram Cytokine Panel for Response to Intravesical Therapy (CyPRIT) $(9,10)$. Recently, the analysis of urine samples as late as one week after the contact with mycobacteria, has also been shown to provide information on the prolonged immune response taking place in the site of the tumor (11). Importantly, the release into urine of very large amounts of soluble factors like CXCL10 $(>250 \mathrm{pg} / \mathrm{mL}$ ) accompanied by high levels of IL-6 and IL-8 (>200 and $>400 \mathrm{pg} / \mathrm{mL}$ respectively), was detected in those patients that had to interrupt BCG therapy, e.g., due to discomfort, suggesting that an excessive inflammatory response might be detrimental for the patients' ability to complete the full course of treatment. These recent observations might imply that devising experimental conditions to stimulate the highest possible immune response might not necessarily be optimal for patients and needs to be balanced with the ability of the patients to tolerate this therapy. More information is still needed to establish good soluble biomarkers in urine, nonetheless, recent efforts are probably closer to help establishing novel follow-up analyses of patients and semi-personalized tools for prognosis.

The exact mechanism of action of BCG to eliminate bladder cancer is not completely understood. Besides the soluble factors found in urine of BCG-treated bladder cancer patients, several immune cell populations infiltrate the bladder after instillations $(12,13)$. Neutrophils and monocytes are recruited to the bladder in large amounts after BCG instillations and different types of effector cells, mainly natural killer (NK) cells and NKT cells are involved in direct elimination of bladder cancer cells (14).

Another aspect studied in the paper by Rodriguez et al. (2) was the degree of activation of CD4 T cells, $48 \mathrm{~h}$ after co-incubation with BCG shown by the expression of the activation markers CD25 and CD69. However, there was heterogeneity in the percentage of activated $\mathrm{CD}^{+}$ $\mathrm{T}$ cells among different donors and this measurement represents only one the various immune effector cascades that could be involved in elimination of the tumour. In fact, the data from Rodriguez et al. show that CD4 stimulation does not correlate with cytokine production: BCG was more potent activating $\mathrm{CD}^{+} \mathrm{T}$ cells than the engineered mycobacteria. In contrast, as mentioned above, cytokine production was higher in response to the recombinant BCG-S1PT than to the wt BCG, thus other cell populations must be contributing to the overall response against mycobacteria. While this type of study confirms that the incubation with mycobacteria and, in particular with engineered mycobacteria can stimulate different arms of the immune system, the effector cells contributing to the killing of bladder cells were not identified in the cytotoxicity assays reported in this system. Data from model systems using experimental animals demonstrated that antigen presentation is not the main mechanism for bladder cancer elimination in the context of BCG (15). Importantly, since the beginning of the use of BCG therapy against bladder cancer, various lines of evidence have suggested the involvement of the innate immune system and, in particular, of NK cells: initially experiments using mice, revealed that NK cells participate actively in the elimination of the tumour $(16,17)$. Later, this idea was also demonstrated in vitro in many complementary research reports $(14,18,19)$. A population of $\mathrm{CD}^{+}$lymphocytes expressing $\mathrm{NK}$ receptors has also been suggested to participate in the elimination of bladder cancer in the context of BCG activation of DCs by mediation of NK receptors.

In all these examples, NKG2D-ligands expressed in tumour cells were crucial for the recognition of bladder cancer (20-22). Recently, an NK subset specialized in the recognition of tumours has been demonstrated to become activated after incubation with BCG in vitro (23). Thus, more clinical research on the role of the innate immune response in bladder cancer treated patients seems of crucial importance in order to profit from the effect of BCG 
on innate immunity. Although considerable laboratorybased research data has been produced, translation of these ideas into the clinic is, in general, scarce. Communication between basic and clinical researchers is paramount to confirm those hypotheses consistently generated by several independent non-clinical laboratories. Many efforts on understanding the mechanism of action of BCG against bladder cancer and on improving the efficacy of this treatment are focussed on theoretical aspects to improve patient response and lower BCG toxicity. In addition, scientists are trying to overcome worldwide episodes of BCG shortage due to manufacturing problems (24) at the same time that other studies focussing on the improvement of a vaccine for tuberculosis are taking place (25). Thus, we should all get together to achieve that bladder cancer patients benefit from all the research avenues with conclusive data, including that on more effective BCG preparations or on the design of non-pathogenic MTB strains as new therapeutic agents.

\section{Acknowledgments}

Funding: Research in the author's laboratory is funded by the Spanish Ministry of Science and Innovation [RTI2018-093569-B100, RTC-2017-6379-1 (MCIU/ AEI/FEDER, EU)] and Madrid Regional Government [IMMUNOTHERCAN-CM (B2017/BMD-3733)/ FEDER]. The author would like to thank all the members from the laboratory for discussions and Dr. Hugh Reyburn for critically reading the manuscript.

\section{Footnote}

Conflicts of Interest: The author has no conflicts of interest to declare.

Ethical Statement: The author is accountable for all aspects of the work in ensuring that questions related to the accuracy or integrity of any part of the work are appropriately investigated and resolved.

\section{References}

1. D'Andrea D, Gontero P, Shariat SF, et al. Intravesical bacillus Calmette-Guerin for bladder cancer: are all the strains equal? Transl Androl Urol 2019;8:85-93.

2. Rodriguez $\mathrm{D}$, Goulart $\mathrm{C}$, Pagliarone $\mathrm{AC}$, et al. In vitro Evidence of Human Immune Responsiveness Shows the
Improved Potential of a Recombinant BCG Strain for Bladder Cancer Treatment. Front Immunol 2019;10:1460.

3. Bisiaux A, Thiounn N, Timsit MO, et al. Molecular analyte profiling of the early events and tissue conditioning following intravesical bacillus calmette-guerin therapy in patients with superficial bladder cancer. J Urol 2009; 181:1571-80.

4. Zuiverloon TC, Nieuweboer AJ, Vekony H, et al. Markers predicting response to bacillus Calmette-Guerin immunotherapy in high-risk bladder cancer patients: a systematic review. Eur Urol 2012;61:128-45.

5. Urquidi V, Goodison S, Kim J, et al. Vascular endothelial growth factor, carbonic anhydrase 9 , and angiogenin as urinary biomarkers for bladder cancer detection. Urology 2012;79:1185 e1-6.

6. de Boer EC, Somogyi L, de Ruiter GJ, et al. Role of interleukin-8 in onset of the immune response in intravesical BCG therapy for superficial bladder cancer. Urol Res 1997;25:31-4.

7. Thalmann GN, Sermier A, Rentsch C, et al. Urinary Interleukin-8 and 18 predict the response of superficial bladder cancer to intravesical therapy with bacillus Calmette-Guerin. J Urol 2000;164:2129-33.

8. Qu K, Gu J, Ye Y, et al. High baseline levels of interleukin-8 in leukocytes and urine predict tumor recurrence in non-muscle invasive bladder cancer patients receiving bacillus Calmette-Guerin therapy: A long-term survival analysis. Oncoimmunology 2017;6:e1265719.

9. Kamat AM, Briggman J, Urbauer DL, et al. Cytokine Panel for Response to Intravesical Therapy (CyPRIT): Nomogram of Changes in Urinary Cytokine Levels Predicts Patient Response to Bacillus Calmette-Guerin. Eur Urol 2016;69:197-200.

10. Gogalic S, Sauer U, Doppler S, et al. Validation of a protein panel for the noninvasive detection of recurrent non-muscle invasive bladder cancer. Biomarkers 2017;22:674-81.

11. Ashiru O, Esteso G, Garcia-Cuesta EM, et al. BCG Therapy of Bladder Cancer Stimulates a Prolonged Release of the Chemoattractant CXCL10 (IP10) in Patient Urine. Cancers (Basel) 2019. doi: 10.3390/ cancers 11070940.

12. Brandau S, Suttmann H. Thirty years of BCG immunotherapy for non-muscle invasive bladder cancer: a success story with room for improvement. Biomed Pharmacother 2007;61:299-305.

13. Kamat AM, Colombel M, Sundi D, et al. BCGunresponsive non-muscle-invasive bladder cancer: 
recommendations from the IBCG. Nat Rev Urol 2017;14:244-55.

14. Brandau S, Riemensberger J, Jacobsen $M$, et al. NK cells are essential for effective BCG immunotherapy. Int J Cancer 2001;92:697-702.

15. Ratliff TL, Ritchey JK, Yuan JJ, et al. T-cell subsets required for intravesical BCG immunotherapy for bladder cancer. J Urol 1993;150:1018-23.

16. Koga S, Taniguchi K, Nishikido M, et al. Development of bacillus Calmette-Guerin-induced antitumor activity in peripheral blood mononuclear cells. Urol Int 1991;47 Suppl 1:80-2.

17. Shapiro A, Ratliff TL, Oakley DM, et al. Reduction of bladder tumor growth in mice treated with intravesical Bacillus Calmette-Guerin and its correlation with Bacillus Calmette-Guerin viability and natural killer cell activity. Cancer Res 1983;43:1611-5.

18. Brandau S, Bohle A. Activation of natural killer cells by Bacillus Calmette-Guerin. Eur Urol 2001;39:518-24.

19. Suttmann H, Jacobsen M, Reiss K, et al. Mechanisms of bacillus Calmette-Guerin mediated natural killer cell activation. J Urol 2004;172:1490-5.

20. Naoe M, Ogawa Y, Takeshita K, et al. Bacillus Calmette-
Guerin-pulsed dendritic cells stimulate natural killer T cells and gammadelta T cells. Int J Urol 2007;14:532-8; discussion 538.

21. García-Cuesta EM, Lopez-Cobo S, Alvarez-Maestro M, et al. NKG2D is a Key Receptor for Recognition of Bladder Cancer Cells by IL-2-Activated NK Cells and BCG Promotes NK Cell Activation. Front Immunol 2015;6:284.

22. Higuchi T, Shimizu M, Owaki A, et al. A possible mechanism of intravesical BCG therapy for human bladder carcinoma: involvement of innate effector cells for the inhibition of tumor growth. Cancer Immunol Immunother 2009;58:1245-55.

23. García-Cuesta EM, Esteso G, Ashiru O, et al. Characterization of a human anti-tumoral NK cell population expanded after BCG treatment of leukocytes. Oncoimmunology 2017;6:e1293212.

24. Cernuschi T, Malvolti S, Nickels E, et al. Bacillus Calmette-Guerin (BCG) vaccine: A global assessment of demand and supply balance. Vaccine 2018;36:498-506.

25. Jeyanathan M, Yao Y, Afkhami S, et al. New Tuberculosis Vaccine Strategies: Taking Aim at Un-Natural Immunity. Trends Immunol 2018;39:419-33.
Cite this article as: Valés-Gómez M. Bacillus Calmette Guérin in bladder cancer: is more immune stimulation better? Transl Androl Urol 2019;8(Suppl 5):S517-S520. doi: 10.21037/ tau.2019.12.23 